\title{
Differential vector competence of Ornithodoros soft ticks for African swine fever virus: What if it involves more than just crossing organic barriers in ticks?
}

Rémi Pereira De Oliveira ${ }^{1,2,3}$, Evelyne Hutet ${ }^{3}$, Renaud Lancelot ${ }^{1,2}$, Frédéric Paboeuf ${ }^{3}$, Maxime Duhayon ${ }^{1,2}$, Fernando Boinas ${ }^{4}$, Adalberto A. Pérez de León ${ }^{5}$, Serhii Filatov ${ }^{6}$, Marie-Frédérique Le Potier ${ }^{3}$ and Laurence Vial ${ }^{1,2^{*}}$

\begin{abstract}
Background: Several species of soft ticks in genus Ornithodoros are known vectors and reservoirs of African swine fever virus (ASFV). However, the underlying mechanisms of vector competence for ASFV across Ornithodoros species remain to be fully understood. To that end, this study compared ASFV replication and dissemination as well as virus vertical transmission to descendants between Ornithodoros moubata, O. erraticus, and O. verrucosus in relation to what is known about the ability of these soft tick species to transmit ASFV to pigs. To mimic the natural situation, a more realistic model was used where soft ticks were exposed to ASFV by allowing them to engorge on viremic pigs.

Methods: Ornithodoros moubata ticks were infected with the ASFV strains Liv13/33 (genotype I) or Georgia2007/1 (genotype II), O. erraticus with OurT88/1 (genotype I) or Georgia2007/1 (genotype II), and O. verrucosus with Ukr12/ Zapo (genotype II), resulting in five different tick-virus pairs. Quantitative PCR (qPCR) assays targeting the VP72 ASFV gene was carried out over several months on crushed ticks to study viral replication kinetics. Viral titration assays were also carried out on crushed ticks 2 months post infection to confirm virus survival in soft ticks. Ticks were dissected. and DNA was individually extracted from the following organs to study ASFV dissemination: intestine, salivary glands, and reproductive organs. DNA extracts from each organ were tested by qPCR. Lastly, larval or first nymph-stage progeny emerging from hatching eggs were tested by qPCR to assess ASFV vertical transmission.
\end{abstract}

Results: Comparative analyses revealed higher rates of ASFV replication and dissemination in O. moubata infected with Liv13/33, while the opposite was observed for O. erraticus infected with Georgia2007/1 and for O. verrucosus with Ukr12/Zapo. Intermediate profiles were found for O. moubata infected with Georgia2007/1 and for O. erraticus with OurT88/1. Vertical transmission occurred efficiently in O. moubata infected with Liv13/33, and at very low rates in O. erraticus infected with OurT88/1.

Conclusions: This study provides molecular data indicating that viral replication and dissemination in Ornithodoros ticks are major mechanisms underlying ASFV horizontal and vertical transmission. However, our results indicate that other determinants beyond viral replication also influence ASFV vector competence. Further research is required to fully understand this process in soft ticks.

Keywords: African swine fever virus, Vector competence, Soft tick, Ornithodoros, Molecular biology

*Correspondence: laurence.vial@cirad.fr

${ }^{1}$ UMR Animals, Health, Territories, Risks and Ecosystems (ASTRE), French

Agricultural Research Center for International Development (CIRAD), Montpellier, France

Full list of author information is available at the end of the article

\section{Background}

African swine fever virus (ASFV) is the etiological agent of African swine fever (ASF), a severe disease of swine that can result in up to $100 \%$ mortality in original author(s) and the source, provide a link to the Creative Commons licence, and indicate if changes were made. The images or other third party material in this article are included in the article's Creative Commons licence, unless indicated otherwise in a credit line to the material. If material is not included in the article's Creative Commons licence and your intended use is not permitted by statutory regulation or exceeds the permitted use, you will need to obtain permission directly from the copyright holder. To view a copy of this licence, visit http://creativecommons.org/licenses/by/4.0/. The Creative Commons Public Domain Dedication waiver (http://creativeco mmons.org/publicdomain/zero/1.0/) applies to the data made available in this article, unless otherwise stated in a credit line to the data. 
populations of domestic pigs and wild boar. Modes of ASFV transmission include pig to pig contact, contaminated food, fomites, and the bite of Ornithodoros soft ticks that are competent vectors $[1,2]$. Vector competence is the ability of an arthropod to acquire, support replication of, and transmit a pathogen to a susceptible vertebrate host. In eastern Africa, ticks of the $O$. moubata group are known vectors and reservoirs of ASFV. In parts of Africa where ASFV is endemic, soft tick vectors enable the persistence of virus transmission within a sylvatic cycle involving warthogs, and occasionally serve as a source of infection for reemergence of the disease in domestic pigs [3]. ASFV was first introduced into Europe, specifically into Portugal and Spain, in 1957 and 1960, respectively. Soft ticks of the $O$. erraticus group were found in several southern regions of those countries where ASF has persisted [4], and vector competence of these soft ticks for local ASFV strains was demonstrated [5-7]. In the 1970s, ASF spread into western Europe, South America, and the Caribbean, but it was successfully eradicated from all those regions, with the exception of the island of Sardinia [8]. In 2007, ASF was re-introduced in Eurasia, spreading from Georgia [9] to the Russian Federation and other countries in eastern and central Europe [10, 11], and further to Asian countries, including China [12]. Quarantine, herd depopulation, and zoning are the only options to eradicate ASF outbreaks and prevent their spread because an efficient vaccine to control ASF remains to be developed [13]. This situation highlights the need to assess the vector competence (including virus reservoir role) of soft ticks established in parts of the world where ASFV is currently present or classified as a high-consequence foreign animal disease with the potential to be introduced [14].

Studies have tested the ability of different Ornithodoros species to transmit diverse ASFV strains. Differences in viral titer and persistence were demonstrated depending on the soft tick and virus strain combination tested [15], which highlights the diversity of factors underlying the competence of Ornithodoros ticks for ASFV, such as the tick species/population or virus strain. For example, in one study $O$. coriaceus was found to be able to maintain the Tengani/62 ASFV strain up to 212 days post infection (PI) [16], but in another study, O. porcinus was able to maintain it for only 133 days [17]. Furthermore, O. coriaceus was found to be able to transmit the Tengani/62 ASFV strain, but not the Uganda/61 $[16,18]$. By comparison, O. porcinus was able to transmit both strains to domestic pigs [17]. These findings emphasize that vector competence was not only related to the tick but also to the ASFV strain. Differences in vector competence were also demonstrated in a recent study where O. moubata transmitted the European ASFV strain Georgia2007/1 to domestic pigs while O. erraticus did not [2]. However, these studies were carried out under different conditions, which makes it difficult to establish robust hypotheses regarding the discrepancies.

Few studies have investigated the replication and dissemination of ASFV within Ornithodoros vectors, especially its spread towards organs potentially involved in ASFV transmission. A study of O. porcinus infected with the Chiredzi/83/1 ASFV strain showed dissemination to the midgut, salivary glands, coxal glands, and reproductive organs [19]. Viral titers were the highest in the salivary glands and the reproductive organs, whereas virus replication was not observed in the nervous tissues of the synganglion, Malpighian tubules, and muscle, and this tick-virus pair was able to transmit ASFV to pigs [19]. Studies testing other soft tick-ASFV associations where transmission was documented also reported virus detection in different organs. For example, transmission was associated with the presence of ASFV in the salivary glands of O. moubata [20], hemolymph and salivary glands of O. turicata [16], or coxal fluid of O. erraticus [5]. The route of virus acquisition also influences the dynamics of infection and transmission. When infecting O. porcinus orally with ASFV strains Pretoriuskop/96/4/1 or Malawi Lil/20/1 using an artificial membrane feeder, virus titers measured in tick homogenates increased by tenfold in 10 days for Pretoriuskop/96/4/1, while declining 1000-fold in 2-6 days for Malawi Lil/20/1. The same Malawi Lil/20/1 ASFV strain inoculated through intrahemocoelic injection was able to replicate in O. porcinus [21]. Taken together, these results suggest that vector competence requires ASFV replication and dissemination in tick vectors to infect organs involved in transmission. However, gaps remain in our understanding of infection dynamics and virus dissemination in Ornithodoros ticks exposed to ASFV strains currently circulating in Eurasia.

To explore underlying mechanisms of ASFV vectorial transmission, in this study we compared the behavior of several strains of the virus in three Ornithodoros species (O. moubata, O. erraticus, and O. verrucosus) by measuring viral replication kinetics, viral titers at given time points, viral dissemination within ticks, and filial infection rates. The effect of the infection on ticks was also assessed. We selected these specific tick-virus pairs since the results of ASFV transmission to pigs have already been published for them [2] and could be linked to the profiles obtained in this present study. Additionally, some of these pairs were considered as positive controls that were naturally observed while the others were representative of the tick-virus 
associations that could possibly be encountered in the current context of ASF emergence in Europe. Our results are discussed with regards to potential determinants of vector competence in soft ticks for ASFV, related to ticks or to the virus. We also identified topics related to the competence of soft ticks as ASFV vectors requiring further investigation.

\section{Methods}

\section{Virus strains}

Four highly virulent ASFV strains were selected. Two of these belong to genotype II: the Georgia2007/1 strain initially isolated in 2007 from a domestic pig originating in Georgia [9], and the Ukr12/Zapo strain isolated in 2012 from a domestic pig in Ukraine [22]. Both are genetically very close [22]. The other two ASFV strains belong to genotype I: the Liv13/33 strain isolated in 1983 from O. moubata inhabiting warthog burrows in Zambia [23], and the OurT88/1 strain isolated in 1988 from O. erraticus collected from pig facilities in Portugal [5]. The Georgia2007/1, Liv13/33, and OurT88/1 strains were kindly provided by Dr. Linda Dixon (OIE reference laboratory, The Pirbright Institute, UK), and the Ukr12/Zapo strain by Dr. Carmina Gallardo (ASF European Union Reference laboratory, CISA-INIA, Valdeolmos, Spain). Viral strains used in this study were amplified on porcine alveolar macrophages, once for strains Liv13/33 and Ukr12/Zapo, and twice for strains Georgia2007/1 and OurT88/1. ASFV was diluted in RPMI medium for intramuscular inoculation of pigs as described previously [2].

\section{Tick species}

Soft ticks of the following species reared in the laboratory were used in this study: (i) O. moubata (s.s.), as described by Bakkes et al. [24], from southern Africa ("Neuchâtel strain, maintained in the Neuchâtel University insectary for at least 20 years and reared at CIRAD Montpellier since 2008); (ii) O. erraticus from Portugal ("Alentejo" strain, collected from the field in 2013 and 2016 and reared at CIRAD Montpellier since that date with 1-5 generations elapsed); and (iii) O. verrucosus from Ukraine (collected from the field in 2014-2015 and reared at the NSC IECVM in Kharkiv, with 1 generation elapsed). All these ticks were maintained at $26{ }^{\circ} \mathrm{C} / 80-90 \%$ relative humidity, as recommended for these species [25].

\section{Tick infection on viremic domestic pigs}

Twenty-two Specific Pathogen-Free (SPF) pigs, split into four independent groups, were each intramuscularly inoculated with a $10^{4}$ hemadsorbing dose $50 \%\left(\mathrm{HAD}_{50}\right)$ of one of the different viral strains listed above. When the pigs became viremic between 3 and 4 days post inoculation, ticks were infected by feeding on them. Petri dishes containing ticks enclosed with a mosquito net were kept on each pig's lower abdomen with a bandage to allow tick feeding through the mesh for $3 \mathrm{~h}$. On the day of tick exposure to ASFV via feeding, pig viremia was quantified by titration; viremia was found to range from $10^{7.5}$ $\mathrm{HAD}_{50}$ to $10^{8.25} \mathrm{HAD}_{50} / \mathrm{ml}$. Five tick-virus combinations were obtained: O. moubata-Liv13/33 (OmL), O. moubata-Georgia2007/1 (OmG), O. erraticus-OurT88/1 (OeO), O. erraticus-Georgia2007/1 (OeG), and O. verrucosus-Ukr12/Zapo (OvZ).

\section{Tick sample processing}

After the infectious blood meal, ticks were sorted into pools dedicated to these analyses: (i) monitoring of ASFV replication in ticks using quantitative real-time $P C R$ (qPCR) on tick homogenates; (ii) estimation of the viral load in ticks using virus titration; (iii) localization of the virus in ticks using qPCR on internal organs after dissection; and (iv) assessment of ASFV vertical transmission by analyzing descendants of all the tick-virus pairs by qPCR. Localization assays could not be carried out for OvZ, due to a lack of material. The number and the stage of ticks used for assays (i) and (ii) are shown in Additional file 1: Table S1. The overall experimental design is shown in Fig. 1. Ticks were individually crushed in phosphate buffered saline solution (PBS) as previously described [2] and stored at $-80{ }^{\circ} \mathrm{C}$ for further analysis. For the kinetic study, tick homogenates were assayed at $0,1,2$, and 3 months PI for all the tick-virus pairs. Additional assays were performed at 8,10 , and 12 months $\mathrm{PI}$ for $\mathrm{OeO}$ and OeG, and at 13 months PI for OmG and OvZ. No additional time points were added for $\mathrm{OmL}$ due to the high mortality in this tick-virus pair (see Results section). For virus titration, the same tick homogenates were assayed 2 months PI for all the tick-virus pairs. Additional assays were carried out at 3 months PI for OmL, at 8 months PI for $\mathrm{OeO}$ and $\mathrm{OeG}$, and at 13 months PI for OmG. The measure at 0 month PI determines the initial amount of virus ingested by individual ticks.

For ASFV localization, ticks were dissected using a sterile scalpel blade and surgical pliers to obtain samples of intestine, salivary glands, and reproductive organs. Dissected organs were stored at $-80{ }^{\circ} \mathrm{C}$ in $200 \mu \mathrm{l}$ of PBS until tested. The dissections of specimens representing all tick-virus pairs were carried out at 10 months PI, and at 3 months PI for OeO and OeG as an additional time point. At 10 months PI, five male ticks and five female ticks were dissected for OeG and OmG, nine females and one male for $\mathrm{OeO}$, and three females and two males for OmL. At 3 months PI, five female and five male ticks were dissected for OeG and OeO.

To test vertical transmission to descendants, i.e., filial infection, we used the first progeny cohort produced 


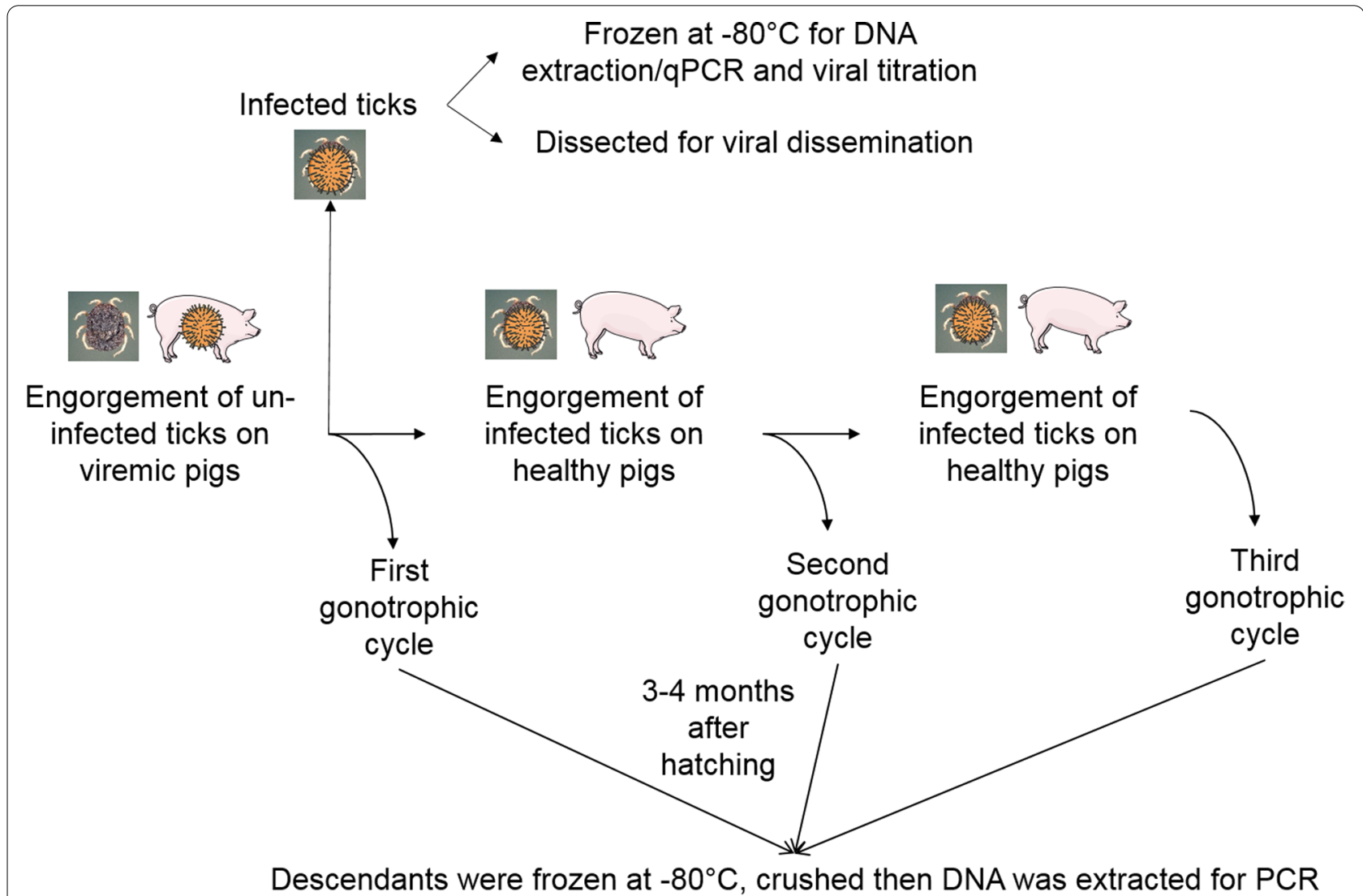

Fig. 1 Depiction of the experimental design used to study the development of African swine fever virus (ASFV) in the infected Ornithodoros soft ticks

following the initial feeding on viremic pigs by female ticks sampled for this purpose. Second and third cohorts of progeny were obtained after the females fed once or twice more on healthy pigs. The third cohort was only available for OmL, OmG, and OeG. Descendants from the first gonotrophic cycle were not kept to be tested for $\mathrm{OeO}, \mathrm{OeG}$, and OvZ. The third cohort of progeny was unavailable for $\mathrm{OeO}$ and OvZ because these ticks were not engorged repeatedly. Only the second cohort was available for these two tick-virus combinations. Tick progeny were tested for ASFV soon after egg hatching, namely larvae for $O$. erraticus and $O$. verrucosus, and the first nymphal stage for O. moubata, since larvae of this latter species molt directly to the next developmental stage without blood-feeding [26]. Descendants were frozen at $-80{ }^{\circ} \mathrm{C}$ between 3 and 4 months after hatching and stored at $-80{ }^{\circ} \mathrm{C}$ until processed. Descendants of OmL and OmG were crushed in $1 \mathrm{ml}$ of PBS, whereas OeO, OeG and OvZ were crushed in $200 \mu$ of PBS due to their smaller size. Initial screenings were done using pools of 20 ticks crushed together. Individual detection was carried out only on a few individuals (8-32) for some tick-virus pairs that did not transmit ASFV vertically.
For tick-virus pairs testing positive in the initial screening, 38 to 206 descendants were tested individually to estimate filial infection rates.

As individuals were kept for several months to monitor ASFV kinetics in ticks and ASFV vertical transmission, it was possible to detect potential extra tick mortality due to ASFV infection. However, we did not check tick survival at regular and predefined time points. Thus, an estimated mortality rate for each tickvirus pair was obtained at 16.5 months PI when the experiment ended. Mortality rates were considered to be high when they reached $5 \%$, which is the maximum natural mortality recorded for our $O$. moubata tick colony in the absence of treatment [27]. The same threshold was applied to the other Ornithodoros species tested because reference values were unavailable in the scientific literature.

\section{Viral genome detection in whole ticks and tick organs}

From ticks used to monitor viral replication or from the progeny used to test vertical transmission, DNA was extracted from $200 \mu \mathrm{l}$ of each crushed tick supernatant using the High Pure PCR Template Preparation Kit 
(Roche Life Science, Penzberg, Germany). Primers and probes detecting the VP72 ASFV gene and the tick betaactin gene were used for duplex qPCR as described previously [2]. For absolute quantification, a plasmid encoding the VP72 and tick beta-actin genes was used at different dilutions to obtain a standard curve and determine the number of gene copies in each sample (Additional file 2: Figure S1).

The qPCR described above was used to assay dissected tick organs. Organs were lysed using tissue lysis buffer according to the manufacturer's procedure (High Pure PCR Template Preparation Kit; Roche Life Science); DNA was extracted using the same kit (Roche Life Science). As we did not monitor ASFV replication in organs and just needed an estimation of the genome viral load in organs at a given time, amplification results were only expressed as the quantification cycle $(\mathrm{Cq})$, i.e., the number of amplification cycles required for the fluorescent signal to cross the detection threshold. A low Cq indicates a large quantity of DNA, while a high Cq indicates a small quantity of DNA.

\section{Virus titration}

Tick homogenates were centrifuged at $10,000 \mathrm{~g}$ for $5 \mathrm{~min}$. The supernatant was serially diluted in RPMI for viral titration in porcine alveolar macrophages, and pig erythrocytes were added the next day as described previously [28]. Viral titers were expressed as $\mathrm{HAD}_{50} / \mathrm{ml}$.

\section{Statistical analysis}

Experimental data were analyzed using the $\mathrm{R}$ statistical package in the RStudio environment ( $R$ Foundation for Statistical Computing, Vienna, Austria). Throughout the analysis, we used $\alpha=0.05$.

To analyze viral replication, the ratio of the VP72 ASFV gene and tick beta-actin gene copy numbers was calculated assuming that: (i) both genes were present in a single copy per genome; (ii) the ratio between VP72 and beta-actin provided the number of ASFV genomes relative to tick genomes, thus correcting for tick size. The number of beta-actin copies in some samples (1 individual in OeG and 7 in OvZ at 0 month PI) was much lower than for other ticks of the same species and stage, which resulted in a significant but abnormal increase in ratios. These ticks may have died before being frozen, which increased the risks of DNA degradation. The outliers with ratios $>300$ were arbitrarily excluded from the analysis.

However, ratios remained very different between individuals, and also between tick-virus pairs. To put all individuals on the same scale and account for ratios equal to 0 (non-infected ticks), each ratio $x$ was firstly transformed into $\log (1+x)$. Preliminary exploratory data analysis revealed each tick-virus pair had a different mean and dispersion. To facilitate the analysis, data of each tick-virus pair were secondly centered and scaled on their own mean and standard deviation according to the formula:

$$
\frac{\log (1+x)-\operatorname{mean}[\log (1+x)]}{s d[\log (1+x)]}
$$

Thus, after this transformation, all tick-virus pairs had the same mean $(=0)$ and the same standard deviation $(=1)$.

A generalized least squares model model was used to account for heteroscedasticity in residuals for each combination of tick-virus $\times$ PI time. This model provided a slope time coefficient for replication kinetics for each tick-virus pair. These time coefficients were compared to the mean to determine whether differences were significant. As no assumption was made on the effect of soft tick sex/stage on vector competence for ASFV, and our initial exploratory data analysis did not detect differences, we grouped sexes and stages together for the overall analysis.

To compare viral titer 2 months PI and to account for the small number of individuals per tick-virus pair $(\leq 15)$, we used non-parametric tests. A Kruskal-Wallis test was used first to detect overall differences between all tick-virus pairs. Then, pairwise Wilcoxon tests were used to determine which tick-virus pairs had different viral titer.

To analyze viral dissemination in ticks, Cq was categorized into four classes: (i) $<25 \mathrm{Cq}$, corresponding to a high load of the VP72 gene; (ii) 25-35 Cq, for an intermediate load of the VP72 gene; (iii) $35-45 \mathrm{Cq}$, for a low load of the VP72 gene; and, (iv) Negative, for negative samples. To determine if the tick-virus pair or the nature of the organ had an effect on viral spread in ticks, an ordinal logistic regression model was used, with the proportion of ticks for each modality as the explanatory variable. OeG was chosen as the reference class for the "tick-virus pair" variable, and salivary glands were the reference for the "organs" variable. A likelihood ratio test was used to assess the effect of variables on the deviance of the model.

A multiple correspondence analysis was applied to detect correlations between the different biological parameters measured in ticks, and the tick-virus pairs that were characterized by distinct abilities to transmit ASFV to pigs. This allowed the data to be summarized and projected on a few orthogonal axes chosen to maximize the projected variance. Results were interpreted in terms of Euclidean distance between the categories of virus replication, viral titer, and dissemination in ticks 
for ASFV vector competence. Table 1 summarizes how biological parameters were arranged in three to five categories and linked to four tick-virus pair classes (OmL, OmG, $\mathrm{OeO}$ and $\mathrm{OeG}$ ) for the analysis.

\section{Ethics statement}

Animal experiments were authorized by the French Ministry for Research (project No. 2017062615498464) and approved by the national ethics committee (Authorization No. 11/07/17-3).

\section{Results}

\section{Mortality in ASFV infected ticks}

The tick-virus pairs OmL and OmG showed very high mortality rates (100 and $73 \%$, respectively), while those of OeG, OeO, and OvZ survived to ASFV infection and showed mortality rates of $<5 \%(2.8,2.1$, and $0 \%$, respectively). Deaths in OmL and OmG primarily occurred in ticks dedicated to vertical transmission trials after their second and third blood meals on naive pigs. Ticks used to monitor ASFV replication, viral load, and ASFV dissemination had not been fed since their initial infective blood meal and did not show increased mortality; consequently, mortality was not taken into account in the analysis of these biological parameters.

\section{ASFV replication in ticks}

Replication rates increased significantly for $\mathrm{OmL}$ between 0 and 1 month PI, as well as between 2 and 3 months PI $(P<0.05$ for both), but decreased significantly between 1 and 2 months $(P<0.05)$ (Fig. 2). Conversely, the ratios decreased significantly from 0 to 12 months PI for OeG $(P$ value $<0.05)$ and from 0 to 13 months PI for OvZ $(P<0.05)$ (Fig. 2). For $\mathrm{OeO}$ and OmG, the ratios did not vary significantly in the first 2 months and then decreased significantly from 3 to 12 months PI for $\mathrm{OeO}(P$ value $<0.05)$ and between 3 and 13 months for OmG $(P<0.05)$ (Fig. 2$)$.

\section{Viral load in ticks}

At 2 months PI, ASFV was isolated from 6/15 ticks in the tick-virus combination OeG, from 14/15 ticks in OeO, from 13/15 ticks in OmG, and from 15/15 in OmL; no viral particles were isolated from ticks in OvZ $(0 / 10)$. With a $P$ value of $3.69 \mathrm{e}-14$, the Kruskal-Wallis test indicated significant differences in virus titer between the tick-virus pairs. Using the pairwise Wilcoxon test, viral titers were not significantly different between the tick-virus combination OeG and OvZ $(P=0.05687)$ or between $\mathrm{OeO}$ and OmG $(P=0.54571)$, with zero to low viral loads in OvZ and OeG $\left(<10^{2.1} \mathrm{HAD}_{50} / \mathrm{ml}\right)$ and intermediate to high viral loads in OeO and OmG $\left(<10^{5.8}\right.$

Table 1 Description of biological parameters used for correspondence analysis

\begin{tabular}{|c|c|c|c|}
\hline Biological parameter & $\begin{array}{l}\text { Name of biological parameter used } \\
\text { in analysis }\end{array}$ & Method used to obtain results & Categories \\
\hline \multirow[t]{4}{*}{ Intestine } & intestine_neg & qPCR & Negative ASFV PCR \\
\hline & intestine_1 & & Cq between 45 and 35 \\
\hline & intestine_2 & & Cq between 35 and 25 \\
\hline & intestine_3 & & $\mathrm{Cq}<\mathrm{r} 25$ \\
\hline \multirow[t]{4}{*}{ Salivary glands } & sg_neg & qPCR & Negative ASFV PCR \\
\hline & sg_1 & & Cq between 45 and 35 \\
\hline & sg_2 & & Cq between 35 and 25 \\
\hline & sg_3 & & $\mathrm{Cq}<25$ \\
\hline \multirow[t]{4}{*}{ Reproductive organs } & ro_neg & qPCR & Negative ASFV PCR \\
\hline & ro_1 & & Cq between 45 and 35 \\
\hline & ro_2 & & Cqt between 35 and 25 \\
\hline & ro_3 & & $\mathrm{Cq}<25$ \\
\hline \multirow[t]{5}{*}{ Viral titer } & titer_neg & Viral titration & Viral titer between $10^{\circ}$ and $10^{2}$ \\
\hline & titer_1 & & Viral titer between $10^{2}$ and $10^{4}$ \\
\hline & titer_2 & & Viral titer between $10^{4}$ and $10^{6}$ \\
\hline & titer_3 & & Viral titer between $10^{\circ}$ and $10^{2}$ \\
\hline & titer_4 & & Viral titer over $10^{6}$ \\
\hline \multirow[t]{3}{*}{ Replication rates } & up_qper & qPCR & Significant increase in viral replication \\
\hline & down_qper & & Significant decrease in viral replication \\
\hline & no_qper & & No significant variation in viral replication \\
\hline
\end{tabular}




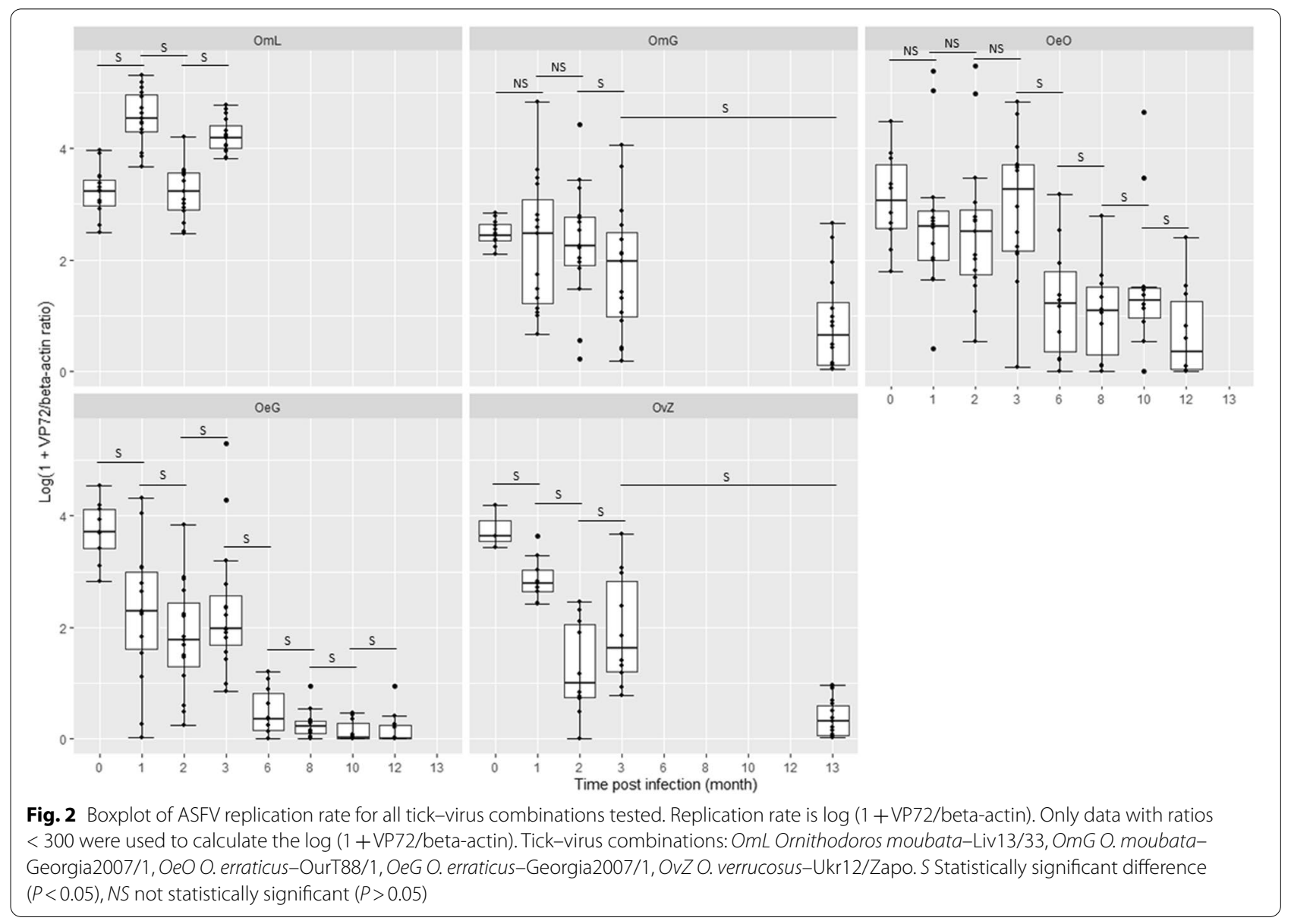

$\mathrm{HAD}_{50} / \mathrm{ml}$ ) (Fig. 3). The differences were significant between other tick-virus combinations $(P<0.05)$ (Fig. 3). Ticks in tick-virus combination OmL displayed a significantly much higher viral load than in those the other combination groups $\left(>10^{5.8} \mathrm{HAD}_{50} / \mathrm{mL}\right)(P$ value $<0.05)$, with particularly low variance between observations (Fig. 3). At 8 months PI, ASFV could still be isolated from $1 / 10$ ticks in the OeG tick-virus combination $\left(10^{2.1}\right.$ $\left.\mathrm{HAD}_{50} / \mathrm{ml}\right)$, and from $3 / 10$ ticks in OeO $\left(10^{2.1}, 10^{2.8}\right.$, and $10^{3.5} \mathrm{HAD}_{50} / \mathrm{ml}$, respectively), with the viral loads seemingly decreasing in OeO. At 13 months PI, ASFV particles were still isolated from $7 / 16$ ticks in OmG $\left(10^{2.1}-10^{5.5}\right.$ $\mathrm{HAD}_{50} / \mathrm{ml}$ ), with also an apparent decrease in viral load, although the difference was not statistically tested. It was not possible to test the long-term persistence of ASFV live particles for the tick-virus combination OmL due to the high mortality observed in OmL ticks as soon as they blood-fed and the necessity to keep the last surviving ticks for the horizontal and vertical transmission trials. At the last titration assay at 3 months PI, ASFV was isolated from 15/15 ticks in OmL, with viral loads similar to those observed at 2 months PI $\left(10^{5.5}-^{6.5} \mathrm{HAD}_{50} / \mathrm{ml}\right)$.

\section{ASFV dissemination in ticks}

The results of ASFV DNA detection in tick organs 10 months PI are shown in Fig. 4. The results for the tick-virus combinations $\mathrm{OeO}$ and $\mathrm{OeG} 3$ months $\mathrm{PI}$ are shown in Fig. 5. At 10 months PI, the ordinal logistic model showed a significant effect of the tick-virus combination $(P<2.2 \mathrm{e}-16)$ and the nature of the organ $(P=0.000729)$ on $\mathrm{Cq}$ levels. Most of the tested organs in the tick- virus combination OmL showed the highest Cq levels, whereas the other tick-virus pairs showed a wide range of $\mathrm{Cq}$ levels in all organs, including also negative results (Fig. 4). OeG was the only tick-virus combination for which $100 \%$ of intestine samples were positive at 10 months PI (Fig. 4). However, as the first barrier encountered by ASFV after an infectious blood meal, the intestine remained the most frequently infected organ in all tick-virus pairs. Regarding the other organs, infected salivary glands were recorded in OmL (100\%), OeO (80\%), OmG (60\%), and then OeG (40\%), the latter being the tick-virus combination with the lowest number of infected salivary glands at 10 months PI (Fig. 4). While the OmL, OmG and OeO tick-virus combinations 


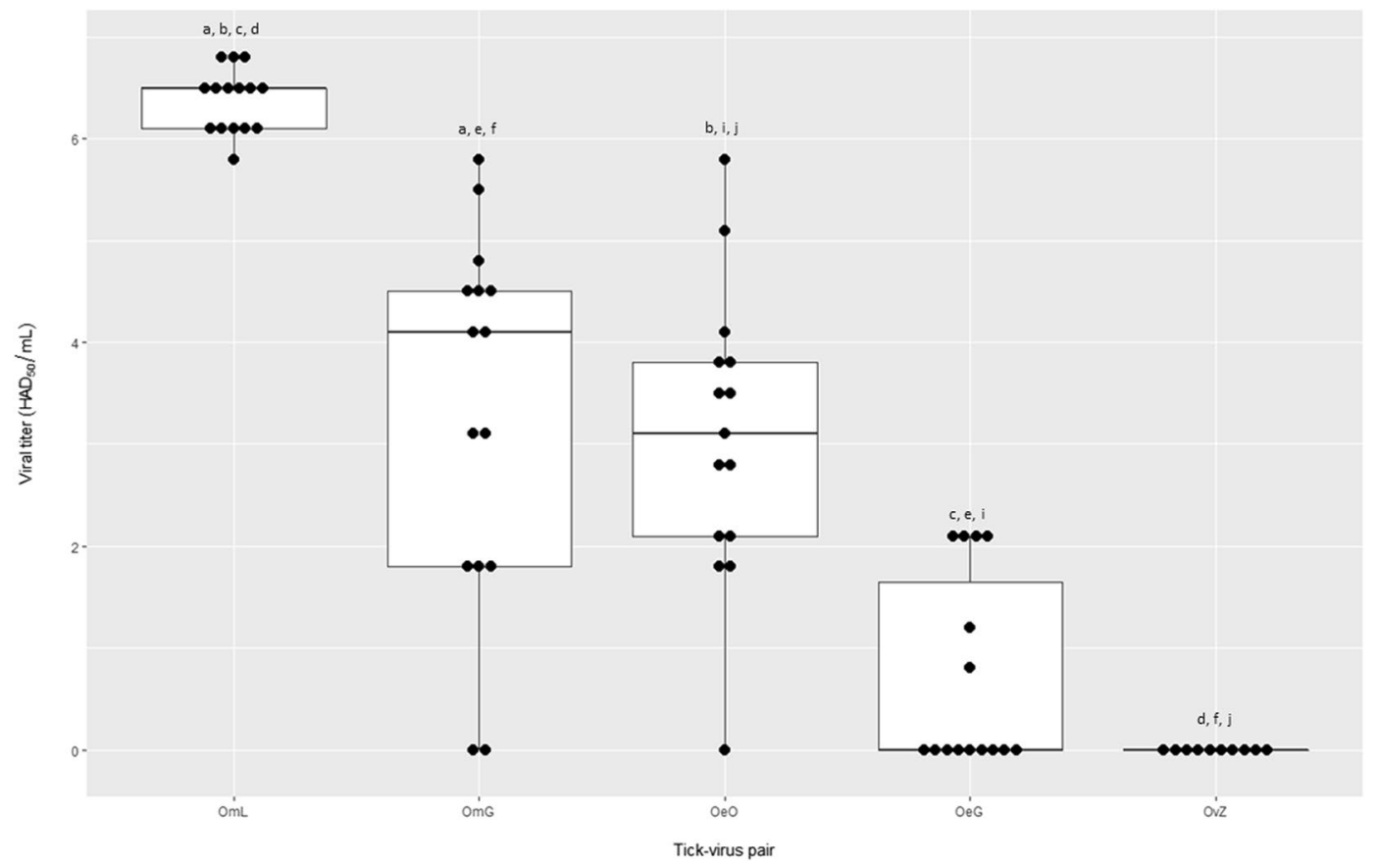

Fig. 3 Boxplot of ASVF titers 2 months post infection for all of the Ornithodoros tick-virus combinations tested. Each black dot corresponds to one tick. Abbreviations for tick-virus combinations tested are as given in Fig. 2 caption. Different lowercase letters indicate significant statistical differences $(P<0.05)$ in viral titer between tick-virus combinations. $H A D_{50}$ Hemadsorbing dose $50 \%$

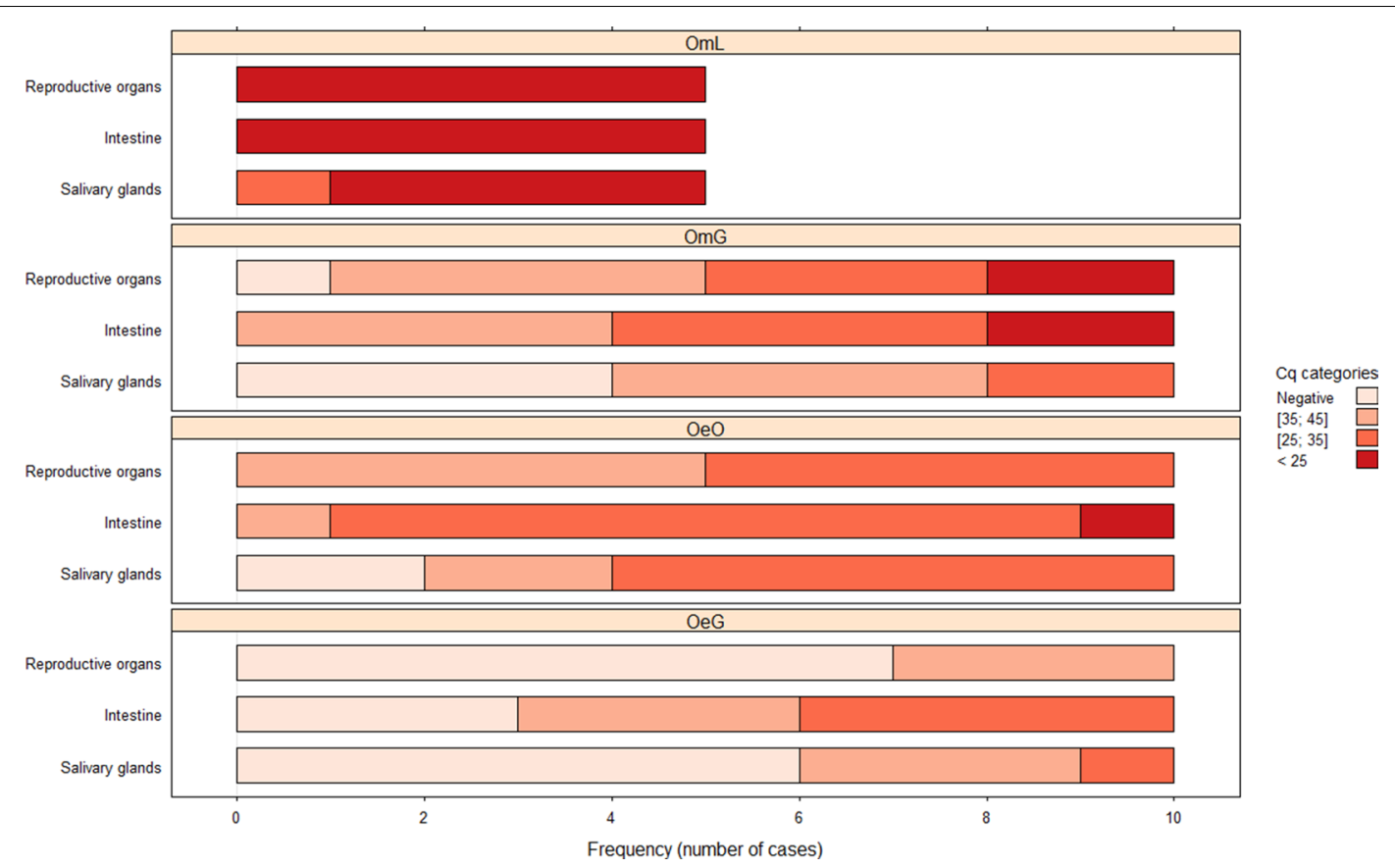

Fig. 4 ASFV localization in organs of soft ticks 10 months post-infection. PCR results, expressed as the quantification cycle (Cq) were split into four categories: (i) Negative organs, when no ASFV genome was detected; (ii) 35-45 Cq, for organs with a low load; (iii) 25-35 Cq, for an intermediate load; and, (iv) <25 Cq, for a high ASFV genome load. Three organs were analyzed: reproductive organs, intestine, and salivary glands. The abbreviations for tick-virus combinations tested are as given in Fig. 2 caption 


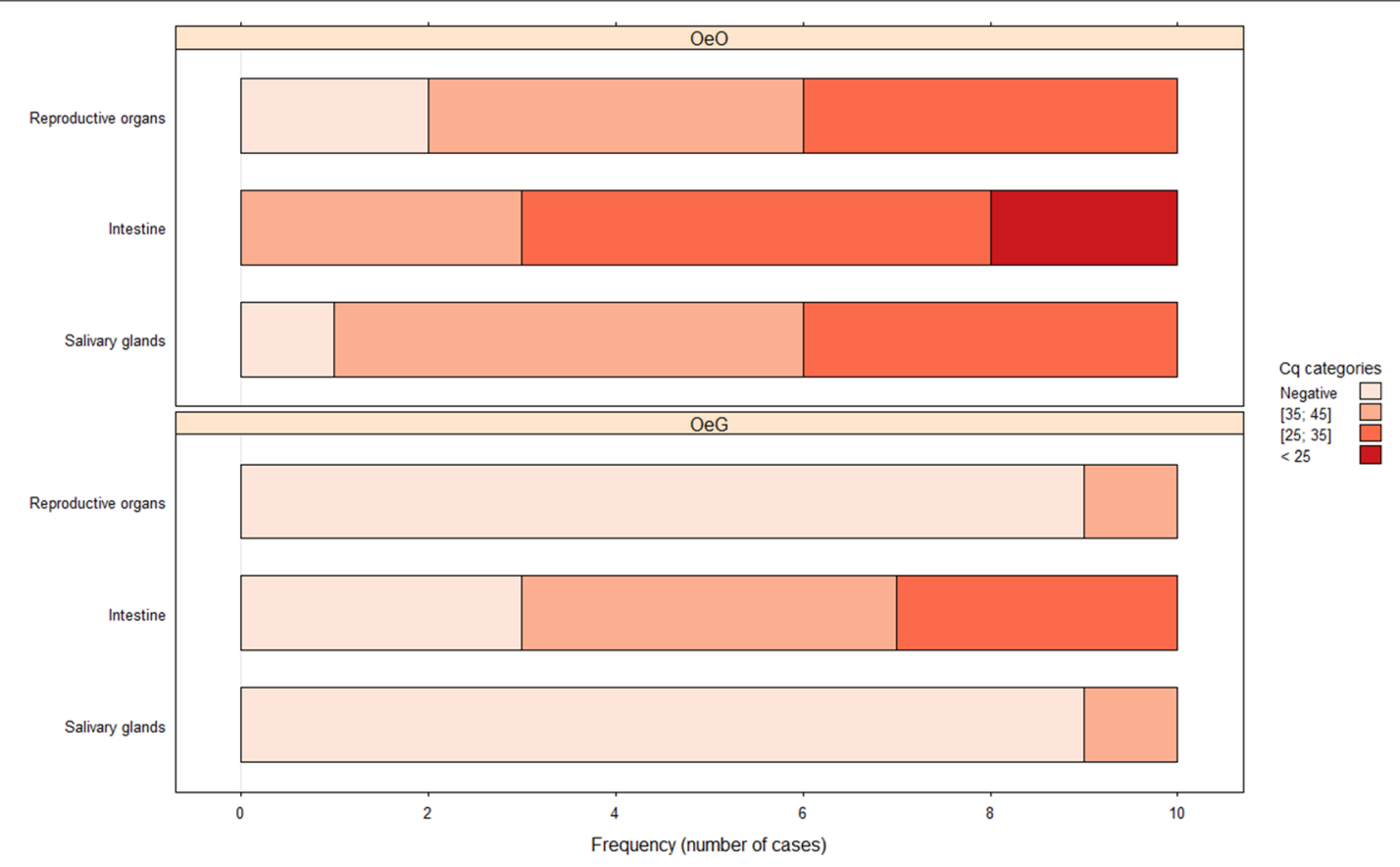

Fig. 5 ASFV localization in infected soft ticks 3 months post-infection. PCR results, expressed as Cq) were split into four categories: (i) Negative organs, when no ASFV genome was detected; (ii) $35-45$ Cq, for organs with a low load; (iii) $25-35$ Cq, for an intermediate load; and (iv) $<25$ Cq for a high ASFV genome load. Three organs were analyzed: reproductive organs, intestine, and salivary glands. The abbreviations for tick-virus combinations tested are as given in Fig. 2 caption

showed $90-100 \%$ positivity of reproductive organs, OeG showed only $30 \%$ of infected reproductive organs at 10 months PI (Fig. 4). Similar results were observed at 3 months PI for the OeO and OeG tick-virus combinations (Fig. 5).

\section{Filial infection with ASFV}

All the tested progeny of OmG, OeG and OvZ were ASFV negative. For OeO, only a single specimen from the second gonotrophic cycle was positive. This corresponded to a filial infection rate of $0.48 \%$ (Table 2). For OmL, positive descendants were detected in every tested cohort. The proportion of infected individuals increased from $9.4 \%$ to $65.8 \%$ and $69.4 \%$ for the first, second, and third gonotrophic cycles, respectively) (Table 2).

\section{Correlation between biological parameters measured in ticks and transmission ability}

Multiple correspondence analysis defined two main synthetic axes explaining $65.2 \%$ (dimension 1) and $25.6 \%$ (dimension 2) of data variance, respectively. Dimension 3 explained only $9.2 \%$ of data variance. The tick-virus combination OmL, characterized by a full transmission capacity, contributed mostly to dimension 1, and OeG, which was not able to transmit in any cases, greatly contributed to dimension 2 . The intermediate virus-tick
Table 2 Vertical African swine fever virus transmission in each tick-virus combination tested

\begin{tabular}{llll}
\hline Gonotrophic cycle & tick-virus pair $^{\mathrm{a}}$ & Treatment & Filial infection rates \\
\hline First & (\%) \\
& OmL & Individual & $10 / 106(9.4 \%)$ \\
& Pool & $6 / 10(60 \%)$ \\
Second & OmL & Individual & $0 / 8$ \\
& OmG & Individual & $25 / 38(65.8 \%)$ \\
& & Individual & $0 / 32$ \\
& OeO & Pool & $0 / 5$ \\
& OeG & Individual & $1 / 206(0.48 \%)$ \\
& & Pool & $0 / 10$ \\
Third & OvZ & Pool & $0 / 1$ \\
& OmL & Individual & $25 / 36(69.4 \%)$ \\
& OmG & Individual & $0 / 8$ \\
& & Pool & $0 / 4$ \\
& OeG & Individual & $0 / 9$ \\
& & Pool & $0 / 10$
\end{tabular}

a OmL, Ornithodoros moubata-Liv13/33; OmG, O. moubata-Georgia2007/1; OeO, O. erraticus-OurT88/1; OeG, O. erraticus-Georgia2007/1; OvZ, O. verrucosusUkr12/Zapo

${ }^{\text {b }}$ Positive individual ticks or pools of 20 ticks (Pool) each/total tested

combination OmG, which only transmitted horizontally, mainly contributed to dimension 3 . OeO, which rarely 
transmitted to its descendants was divided between dimensions 2 and 3. Increased ASFV replication rates, high viral titers, and high $\mathrm{Cq}$ values in organs mainly contributed to the first axis and were all correlated to tick-virus combination OmL (Fig. 6). Conversely, decreased ASFV replication rates, low to zero viral titers, and high Cq values in organs mainly contributed to axis 2 and were correlated to OeG (Fig. 6). OmG and OeO were associated with intermediate ASFV replication and dissemination patterns, which contributed to both the second and third axes (Fig. 6).

\section{Discussion}

This study highlighted differences in the kinetics of ASFV replication and dissemination inside Ornithodoros vectors. The results are in agreement with those reported previously showing variation in virus titers and persistence of infection across Ornithodoros species that is dependent on the ASFV strain used [15]. On the one side of the variability spectrum we observed that the OmL tick-virus combination resulted in ASFV transmitted horizontally and vertically with increasing replication, high viral titers, and efficient virus dissemination to the internal organs. On the other side of the spectrum, ASFV in the OeG tick-virus combination was not transmitted to pigs or to the O. erraticus progeny, virus was cleared over time, and ticks yielded low viral titers with poor organ dissemination. Interestingly, the other two tickASFV combinations displayed intermediate profiles, with OmG transmitting ASFV only horizontally and OeO transmitting only vertically, but with a very low efficiency rate (a single positive tick out of 206 tested). Both of these latter tick-virus combinations showed similar ASFV replication rates, as well as similar viral titers 2 months PI and similar dissemination efficiencies.
The results presented here suggest that events other than the infection of internal organs are required for Ornithodoros ticks to transmit ASFV. In a previous study on ASFV horizontal transmission, we showed that the OmG tick-virus combination resulted in virus transmission when 30 ticks bit a pig at a single time [2], whereas transmission failed when multiple tick challenges were carried out using 15 ticks each time. We thus hypothesized that the proportion of infected soft ticks, and thus the proportion of ticks with infected internal organs, is important factor in the transmission success. Quantitative aspects related to bioactive salivary gland factors may also play a role in the competence of Ornithodoros ticks as ASFV vectors [29]. Furthermore, we showed, using correspondence analysis, that ASFV replication in ticks, as well as the resulting viral loads in particular internal organs, are additional essential factors for explaining transmission success. However, it must be noted that we did not determine viral dissemination in the OvZ tickvirus combination as an insufficient number of ticks were available.

The observation that while O. moubata transmitted ASFV to pigs efficiently they also perished from viral infection suggests that Ornithodoros species differ in their capacity to control ASFV infection. However, this excess mortality does not alter the vector competence of O. moubata as it only occurred after the second blood meal when ASFV was transmitted horizontally and/ or vertically. As mentioned before [15], this stresses the need for research to enhance our understanding of the molecular basis of the interaction between ASFV and soft ticks. In the less effective tick-virus pairs, OeG and OvZ, which did not die from infection, ASFV replication decreased considerably, especially by 2 months PI, which indicates a gradual clearance of the virus from the

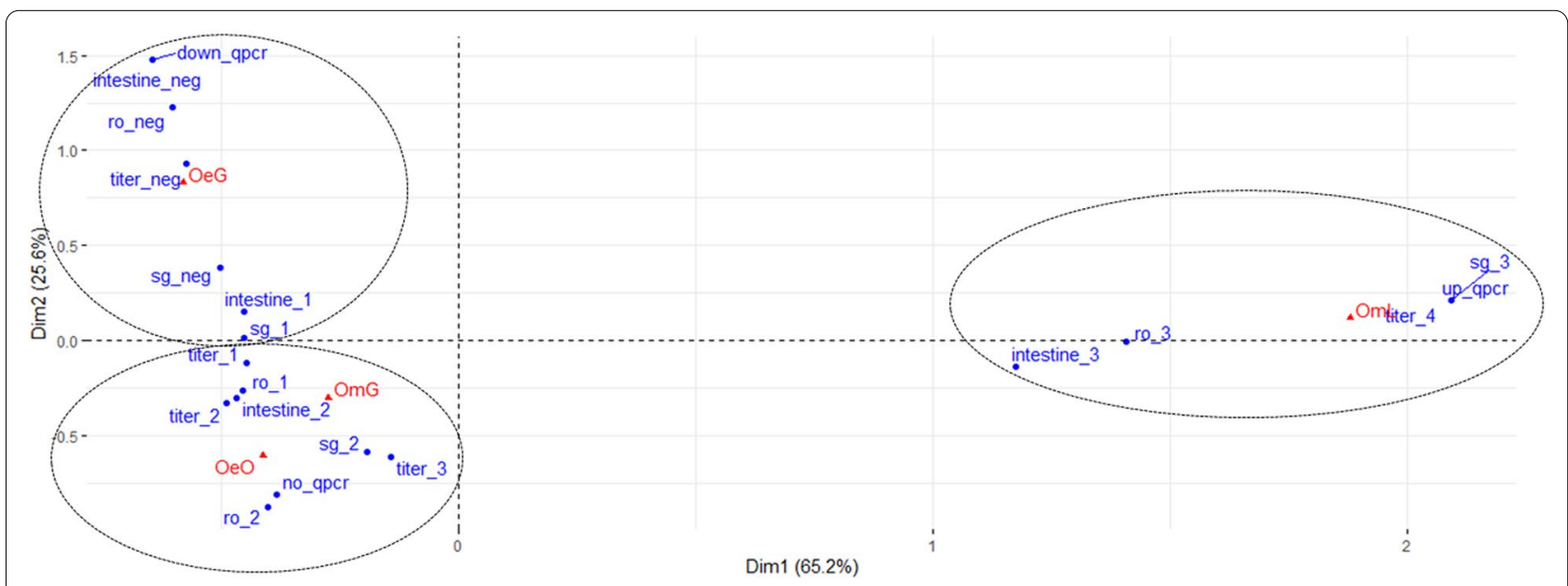

Fig. 6 Bi-dimensional plot showing the correspondence analysis of multiple variables and different tick-virus combinations. Dim Dimension. See section Materials and Methods for details. The third axis is not represented in the bi-dimensional plot 
ticks. However, in another study using the Georgia2007/1 strain and O. erraticus ticks, ASFV replicated very efficiently [30]. The main difference between the results of that and our studies is that the ticks used by Diaz et al. [30] were artificially engorged on blood in the presence of antibiotics and antifungals. This treatment may have directly modified the integrity of the tick midgut, favoring the crossing of ASFV into the hemocoel and therefore bypassing midgut replication (known as the "leaky-midgut" phenomenon) [31]. Another hypothesis is that the presence of such substances in the blood meal could have affected the results because antibiotics are known to alter the microbiome and the metabolism of ticks [27].

Microbiome modifications in arthropod vectors are known to affect the replication of pathogens, including viruses [32-35]. Interestingly, when they were reared in Neuchâtel before being transferred to CIRAD, O. moubata were maintained on blood treated with antibiotics for several years. A study using Aedes aegypti showed shown that a modification of the midgut microbiome had an impact on the basal immune response of mosquitoes by changing the expression of genes involved in the immune response, such as antimicrobial peptides, which in turn impacted Dengue virus replication [34]. In the hard ticks, Ixodes ricinus and Haemaphysalis longicornis, defensins have been shown to have an antimicrobial effect on Gram-positive bacteria [36, 37]. In the soft tick Ornithodoros moubata, a challenge with $E$. coli upregulated expression of the gene coding for defensins $A$ and $B$ in the midgut [38]. It was also reported that soft tick engorgement changed the expression of immune genes, such as the genes coding for antimicrobial peptides, in different soft ticks species (O. moubata, $O$. erraticus and O. turricata) [38-43]; however, the antiviral effect of this changed expression needs to be tested. Among the large panel of immune responses described in arthropods against transmitted pathogens [44], RNA interference is an important immune response against virus infection, having an impact on vector competence [45]. The authors of a recent study suggested that ASFVlike integrated elements coding for small RNA in $O$. moubata could serve to protect ticks from ASFV infection through the RNA interference mechanism [46]. More specifically, the alignment of these small RNAs on the full ASFV genome showed more than 500 small RNAs that matched with ASFV genomes from genotype II (like Georgia2007/1), while there were fewer than 20 matching small RNAs with several ASFV genomes from genotype I. These results might also be an explanation for the differences in ASFV kinetics and mortality rates observed in O. moubata ticks infected with Liv13/33 from genotype I (OmL) versus O. moubata infected with Georgia2007/1 from genotype II (OmG).
Previous studies revealed that some ASFV genes, such as those of the Multigen family 360 (MGF-360) and $\mathrm{CD} 2 \mathrm{v}$ protein, could be involved in viral replication in ticks, with the deletion of these genes having a negative impact on the efficiency of virus replication in the soft ticks O. porcinus and O. erraticus, but not blocking it [47, 48]. Molecular analysis of the viral strains Liv13/33 [49] and Georgia2007/1 [50] used in our experiments showed the existence of these genes in both strains. The genomes of OurT88/1 and Ukr12/Zapo remain unknown, which makes the comparison with these ASFV strains difficult. However, ASFV replication differed between the tick-virus combinations $\mathrm{OmL}$ and $\mathrm{OmG}$, and even the presence of these ASFV genes might not be sufficient to explain differences in ASFV replication in these tickvirus combinations, which raises the possibility that other as yet undetermined viral genes could affect the ability of ASFV to replicate in soft ticks. Other genes may be involved in ASFV replication in ticks. Another comparison between Liv13/33 and Georgia2007/1 highlighted the presence of the X64R gene in Liv13/33 but not in Georgia2007/1, which suggests that this gene could be implicated in viral replication (http://asfvdb.popgenetics. net/) [49]. The X64R gene is also present in most, but not all, strains of genotype I, but has never been reported for genotype II, including the Georgia2007/1 strain (http:// asfvdb.popgenetics.net/). Further research is required to test the hypothesis that the presence of such genes might contribute to the ability of the tick-virus combinations $\mathrm{OmL}$ and $\mathrm{OeO}$ to transmit ASFV vertically, contrary to OmG, OeG, and OvZ as observed in our study. Notably, vertical transmission has been reported with ASFV strains from genotype I $[16,51]$ and in two cases with strains from genotype XX and an undefined genotype $[21,52]$. Strains from genotype II and $X$ failed to be transmitted vertically by ticks $[18,53]$, and in one case transmission also failed with the L60 strain from genotype I [18], although this latter strain has the X64R gene. However, a complete sequence was not available for all the ASFV strains used in these vertical transmission studies, which limits genome comparisons. The possible role of certain viral genes in replication in soft ticks highlights the complexity of ASFV-Ornithodoros interactions underlying vector competence [54].

Noteworthy is the vertical transmission of Liv13/33 in the OmL virus-tick combination to the first progeny after the infectious blood meal. By comparison, Rennie et al. [51] observed vertical transmission in the second gonotrophic cycle with the same soft tick-ASFV combination. To the best of our knowledge, this is the first time that this transmission pattern is described with ASFV $[18,51-53,55,56]$ or any other soft tick-borne pathogen such as Borrelia duttoni [26]. Ornithodoros 
moubata ovogenesis begins 4-5 days after the blood meal, allowing the acquisition of an impermeable membrane that may prevent the infection of eggs, as demonstrated for Borrelia duttoni [26]. Soft ticks lay eggs about 15 days after a blood meal [25]. As it takes an average of 2-3 weeks for ASFV to disseminate inside soft ticks and reach the reproductive organs after the infectious blood meal [19], it seems unlikely for the eggs of the first gonotrophic cycle to become infected. As we ensured that our $O$. moubata ticks were free of AFSV before experimentation, we surmise that ASFV dissemination inside soft ticks resulted in the infection of the latest maturated portion in the egg batch. This is congruent with the observation that $<10 \%$ of descendants in the first gonotrophic cycle were infected while $>65 \%$ were positive in the second and third cycles. Differences between our study and the experiment by Rennie et al. [51] include the method of engorgement and the initial virus titer to infect ticks. Under our experimental conditions, Ornithodoros ticks were infected with ASFV by taking a blood meal on viremic pigs with virus titers of between $10^{7.5}$ and $10^{8.25}$ $\mathrm{HAD}_{50} / \mathrm{ml}$ while Rennie et al. [51] infected ticks with an artificial feeding system using a mix of uninfected blood and viremic blood with a final titer of $10^{5} \mathrm{HAD}_{50} / \mathrm{ml}$. The initial dose might have affected the speed of ASFV dissemination in the ticks and thus the infection of the reproductive organs and transmission to the eggs. In other arbovirus-vector systems, the initial dose is known to affect the speed of virus spread, as shown, for example, with Bluetongue virus in Culicoides sonorensis [57] and for Zika virus in Aedes aegypti [58]. Equally plausible is a higher susceptibility for ASFV infection in the $O$. moubata we used that have been reared under laboratory conditions for $\sim 25$ years relative to the conspecific yet genetically distinct ticks used by Rennie et al. [51]. Keeping O. moubata as a closed colony in the laboratory for that period of time may have rendered the ticks highly inbred with fixed alleles, and a poor microbiome receiving only blood from healthy animals would limit exposure to a diverse micro-organisms, resulting in a poorly stimulated or even immunologically naive immune system compared to ticks collected directly from the field [59]. It is likely that in the wild, ticks are probably exposed to ASFV multiple times through blood-feeding during their development cycle, which can range from 6 months to several years to complete, depending on host availability and environmental conditions [25]. It is possible that constant exposure to environmental microbes shapes immunological maturity, training the innate immune system and phenotypic susceptibility to a local microbe, as well as inducing trained immunity, all factors providing cross-protection against other pathogens [59].
The interplay between genetic and environmental factors influences how the competence of arthropods as vectors of infectious agents evolves. This needs to be taken into consideration when trying to extrapolate results from laboratory experiments to try to explain what happens in the field. For example, in a previous study, two laboratory colonies of $O$. turicata established using ticks collected at different times and from different zones did not display the same results of vector competence for ASFV, as one was able to transmit the virus to pigs while the other one was not [16]. In this context, the genetic evolution hypothesis may explain why, under our experimental conditions, the tick-virus combination OeO did not transmit ASFV to pigs [2], but on rare occasions did transmit the virus to its descendants. In studies conducted 20 years previously, the same tick species was able to transmit the same strain (OurT88/1) horizontally [5]. The O. erraticus ticks used in these studies were from colonies established with field-collected specimens from the same region, but not the same pig farms and thus they represented different populations. In addition to space, collection times also differed. The colony sourcing the ticks used in the previous study was established with O. erraticus collected sometime 1970s and the 1990s when ASFV was circulating in the region. However, the conspecific ticks used in our study were from a colony started with specimens collected from the same region where ASF had been eradicated for 20 years and, therefore, co-evolutionary forces for the adaptation of ASFV to infect and persist in O. erraticus could have been weakened through time. The same observations have been published for mosquitoes, that is, the local genetic evolution of vector populations may influence pathogen replication, dissemination, and transmission [60-62].

Further studies assessing how the microbiome may influence the vector competence of Ornithodoros ticks for ASFV are needed given the recognition that the microbiota associated with ticks can shape their immunological response to infectious agents and determine the ability to transmit tick-borne pathogens [59, 63]. The application of soft tick genomics will help unveil the molecular basis of soft tick competence for ASFV. This needs to be coupled with the development of genetic markers in Ornithodoros ticks and ASFV to investigate hypotheses of local coevolution.

\section{Conclusion}

The replication and dissemination patterns of ASFV in soft ticks were different between the five tick-virus combinations tested. Replication and 
disseminationwere were shown to be major determinants underlying successful horizontal and vertical transmission of the virus. Factors such as the Ornithodoros immune response against ASFV infection, the genetic background of ASFV and/or soft ticks, and the tick microbiome may also play a major role in vector competence. These research areas merit attention to enhance preparedness against ASF outbreaks where Ornithodoros ticks may be involved as ASFV vectors.

\section{Supplementary Information}

The online version contains supplementary material available at https://doi. org/10.1186/s13071-020-04497-1.

Additional file 1: Table S1. The number and the stage of ticks used for the study.

Additional file 2: Figure S1. Plasmid used for the standard curve for qPCR. Beta-actin primers are shown in cyan and the beta-actin probe in yellow. The ASFV-VP72 gene primers are shown in green and the ASFVVP72 probe in red.

\section{Abbreviations}

ASF: African swine fever; ASFV: African swine fever virus; $\mathrm{HAD}_{50}$ : Hemadsorbing dose 50\%; OeG: Ornithodoros erraticus-Georgia2007/1; OeO: Ornithodoros erraticus-OurT88/1; OmG: Ornithodoros moubata-Georgia2007/1; OmL: Ornithodoros moubata-Liv13/33; SPF: Specific Pathogen-Free.

\section{Acknowledgements}

Thanks to Vectopole Sud for funding the insectary where Ornithodoros moubata and O. erraticus were reared and to the Direction Générale de I'Alimentation for financial support to CIRAD. The authors also thank Angélique Moro and Jean-Marie Guionnet for animal care at ANSES, Olivier Duron for providing the plasmid used for DNA quantification, and Peter Biggins for English editing.

\section{Authors' contributions}

LV and MFLP conceived and designed the study. LV supervised the study. RPDO conceived the study, performed the laboratory experiments, and wrote the draft of the manuscript. EH performed the laboratory experiments. RL and RPDO analyzed the data. FP provided domestic pigs. FB provided Ornithodoros erraticus. SF provided O. verrucosus. AAPL contributed to the establishment of the $O$. verrucosus colony using field specimens collected in Ukraine and edited the manuscript. All authors reviewed the manuscript and read and approved the final manuscript.

\section{Funding}

This study was partially funded by the NSF-NIH-EEID ASF project (Grant No. 2019-67015-28981). The assessment of the study of O. verrucosus was made possible by the financial support of the U.S. Defense Threat Reduction Agency grant [CBEP Agreement IAA\# U.S.C. 3318(b)-15217] involving Adalberto A. Pérez de León and a Short Term Scientific Mission Grant for Serhii Filatov from COST Action CA15116. The PhD grant of Rémi Pereira De Oliveira was funded by CIRAD and ANSES.

\section{Availability of data and materials}

All data generated or analyzed during this study are included in this published article.

\section{Ethics approval and consent to participate}

The animal experiments were authorized by the French Ministry for Research (project No. 2017062615498464) and approved by the national ethics committee (authorization No. 11/07/17-3).

\section{Consent for publication}

Not applicable.

\section{Competing interest}

The authors declare that they have no competing interests.

\section{Author details}

${ }^{1}$ UMR Animals, Health, Territories, Risks and Ecosystems (ASTRE), French Agricultural Research Center for International Development (CIRAD), Montpellier, France. ${ }^{2}$ UMR ASTRE, CIRAD, National Research Institute for Agriculture, Food and the Environment (INRAE), University of Montpellier, Montpellier, France.

${ }^{3}$ Swine Virology and Immunology Unit, Ploufragan-Plouzané-Niort Laboratory, French Agency for Food, Environmental and Occupational Health \& Safety (ANSES), Ploufragan, France. ${ }^{4}$ Center for Interdisciplinary Research in Animal Health (CIISA), Faculty of Veterinary Medicine, University of Lisbon, Avenida da Universidade Técnica, Lisbon 1300-477, Portugal. ${ }^{5}$ Knipling-Bushland U.S. Livestock Insects Research Laboratory and Veterinary Pest Genomics Center, US Department of Agriculture-Agriculture Research Service (USDA-ARS), Kerrville, TX, USA. ${ }^{6}$ National Scientific Center Institute of Experimental and Clinical Veterinary Medicine (NSC IECVM), Kharkiv, Ukraine.

Received: 8 July 2020 Accepted: 23 November 2020

Published online: 09 December 2020

\section{References}

1. O'Neill X, White A, Ruiz-Fons F, Gortázar C. Modelling the transmission and persistence of African swine fever in wild boar in contrasting European scenarios. Sci Rep. 2020;10:5895.

2. Pereira de Oliveira R, Hutet E, Paboeuf F, Duhayon M, Boinas F, Perez de Leon A, et al. Comparative vector competence of the Afrotropical soft tick Ornithodoros moubata and Palearctic species, O. erraticus and O. verrucosus, for African swine fever virus strains circulating in Eurasia. PLoS ONE. 2019;14:e0225657.

3. Penrith M-L, Vosloo W, Jori F, Bastos ADS. African swine fever virus eradication in Africa. Virus Res. 2013;173:228-46.

4. Boinas F, Ribeiro R, Madeira S, Palma M, de Carvalho IL, Núncio S, et al. The medical and veterinary role of Ornithodoros erraticus complex ticks (Acari: Ixodida) on the Iberian Peninsula. J Vector Ecol. 2014;39:238-48.

5. Boinas FJS. The role of Ornithodoros erraticus in the epidemiology of African swine fFever in Portugal. PhD thesis. Reading: University of Reading; 1994.

6. Boinas FS, Wilson AJ, Hutchings GH, Martins C, Dixon LJ. The persistence of African swine fever virus in field-infected Ornithodoros erraticus during the ASF endemic period in Portugal. PLoS ONE. 2011;6:e20383.

7. Caiado JM, Boinas FS, Louza AC. Epidemiological research of African swine fever (ASF) in Portugal: the role of vectors and virus reservoirs. Acta Vet Scand Suppl. 1988;84:136-8.

8. Costard S, Wieland B, de Glanville W, Jori F, Rowlands R, Vosloo W, et al. African swine fever: how can global spread be prevented? Philos Trans R Soc B Biol Sci. 2009;364:2683-96.

9. Rowlands RJ, Michaud V, Heath L, Hutchings G, Oura C, Vosloo W, et al. African swine fever virus isolate, Georgia, 2007. Emerg Infect Dis. 2008;14:1870-4.

10. Chenais E, Depner K, Guberti V, Dietze K, Viltrop A, Ståhl K. Epidemiological considerations on African swine fever in Europe 2014-2018. Porc Health Manag. 2019;5:6.

11. Linden A, Licoppe A, Volpe R, Paternostre J, Lesenfants C, Cassart D, et al. Summer 2018: African swine fever virus hits north-western Europe. Transbound Emerg Dis. 2019;66:54-5.

12. Wen X, He X, Zhang X, Zhang X, Liu L, Guan Y, et al. Genome sequences derived from pig and dried blood pig feed samples provide important insights into the transmission of African swine fever virus in China in 2018. Emerg Microbes Infect. 2019;8:303-6.

13. Arias M, Jurado C, Gallardo C, Fernández-Pinero J, Sánchez-Vizcaíno JM. Gaps in African swine fever: analysis and priorities. Transbound Emerg Dis. 2018:65:235-47. 
14. Brown VR, Bevins SN. A review of African swine fever and the potential for introduction into the United States and the possibility of subsequent establishment in feral wwine and native ticks. Front Vet Sci. 2018;5:11.

15. Burrage TG. African swine fever virus infection in Ornithodoros ticks. Virus Res. 2013;173:131-9.

16. Hess WR, Endris RG, Haslett TM, Monahan MJ, McCoy JP. Potential arthropod vectors of African swine fever virus in North America and the Caribbean basin. Vet Parasitol. 1987;26:145-55.

17. Plowright W, Parker J, Pierce MA. The epizootiology of African swine fever in Africa. Vet Rec. 1969;85:668-74.

18. Groocock CM, Hess WR, Gladney WJ. Experimental transmission of African swine fever virus by Ornithodoros coriaceus, an argasid tick indigenous to the United States. Am J Vet Res. 1980;41:591-4.

19. Kleiboeker SB, Burrage TG, Scoles GA, Fish D, Rock DL. African swine fever virus infection in the argasid host Ornithodoros porcinus porcinus. J Virol. 1998;72:1711-24.

20. Rennie LF. The persistance of African swine fever virus in the argasid tick Ornithodoros moubata. PhD thesis. Hatfield: University of Hertfordshire; 1998

21. Kleiboeker SB, Scoles GA, Burrage TG, Sur J. African swine fever virus replication in the midgut epithelium is required for infection of Ornithodoros ticks. J Virol. 1999;73:8587-98.

22. Gallardo C, Fernández-Pinero J, Pelayo V, Gazaev I, Markowska-Daniel I, Pridotkas $\mathrm{G}$, et al. Genetic variation among African swine fever genotype II viruses, eastern and central Europe. Emerg Infect Dis. 2014;20:1544-7.

23. Dixon LK, Wilkinson PJ. Genetic diversity of African swine fever virus isolates from soft ticks (Ornithodoros moubata) inhabiting warthog burrows in Zambia. J Gen Virol. 1988;69:2981-93.

24. Bakkes DK, De Klerk D, Latif AA, Mans BJ. Integrative taxonomy of Afrotropical Ornithodoros (Ornithodoros) (Acari: Ixodida: Argasidae). Ticks Tick Borne Dis. 2018;9:1006-37.

25. Vial L. Biological and ecological characteristics of soft ticks (lxodida: Argasidae) and their impact for predicting tick and associated disease distribution. Parasite. 2009;16:191-202.

26. Aeschlimann A. Développement embryonnaire d'Ornithodoros moubata (Murray) et transmission transovarienne de Borrelia duttoni. Acta Trop. 1958; 15(1):5-64.

27. Duron O, Morel O, Noël V, Buysse M, Binetruy F, Lancelot R, et al. Tickbacteria mutualism depends on B vitamin synthesis pathways. Curr Biol. 2018;28(1896-1902):e5.

28. Carrascosa AL, Bustos MJ, de Leon P. Methods for growing and titrating African swine fever virus: field and laboratory samples. Curr Protoc Cell Biol. 2011; Chapter 26:Unit 26.14.

29. Bernard J. Ornithodoros tick vector competence characteriation for African swine fever virus and study of two vector competence determinants: virus strain-vector relationship and tick saliva influence on domestic pig infection. PhD thesis. Montpellier: University of Montpellier; 2015.

30. Diaz AV, Netherton CL, Dixon LK, Wilson AJ. African swine fever virus strain Georgia 2007/1 in Ornithodoros erraticus ticks. Emerg Infect Dis. 2012;18:1026-8.

31. Janeh M, Osman D, Kambris Z. Comparative analysis of midgut regeneration capacity and resistance to oral infection in three disease-vector mosquitoes. Sci Rep. 2019:9:14556.

32. Meister S, Agianian B, Turlure F, Relógio A, Morlais I, Kafatos FC, et al. Anopheles gambiae PGRPLCmediated defense against bacteria modulates infections with malaria parasites. PLoS Pathog. 2009;5:e1000542.

33. Mukherjee D, Das S, Begum F, Mal S, Ray U. The mosquito immune system and the life of dengue virus: what we know and do not know. Pathogens. 2019;8:77.

34. Ramirez JL, Souza-Neto J, Torres Cosme R, Rovira J, Ortiz A, Pascale JM, et al. Reciprocal tripartite interactions between the Aedes aegypti midgut microbiota, innate immune system and Dengue virus influences vector competence. PLoS Negl Trop Dis. 2012;6:e1561.

35. Rodgers FH, Gendrin M, Wyer CAS, Christophides GK. Microbiota-induced peritrophic matrix regulates midgut homeostasis and prevents systemic infection of malaria vector mosquitoes. PLOS Pathog. 2017;13:e1006391.

36. Chrudimská T, Slaninová J, Rudenko N, Růžek D, Grubhoffer L. Functional characterization of two defensin isoforms of the hard tick /xodes ricinus. Parasites Vectors. 2011:4:63.
37. Yada Y, Talactac MR, Kusakisako K, Hernandez EP, Galay RL, Andoh M, et al. Hemolymph defensin from the hard tick Haemaphysalis longicornis attacks Gram-positive bacteria. J Invertebr Pathol. 2018;156:14-8.

38. Nakajima Y, van Naters-Yasui A van der G, Taylor D, Yamakawa M. Two isoforms of a member of the arthropod defensin family from the soft tick, Ornithodoros moubata (Acari: Argasidae). Insect Biochem Mol Biol. 2001;31:747-51.

39. Armstrong BA, Kneubehl AR, Mitchell RD, Krishnavajhala A, Teel PD, Pérez de León AA, et al. Differential expression of putative Ornithodoros turicata defensins mediated by tick feeding. Front Cell Infect Microbiol. 2020;10:152.

40. Grunclová L, Fouquier H, Hypša V, Kopáček P. Lysozyme from the gut of the soft tick Ornithodoros moubata: the sequence, phylogeny and postfeeding regulation. Dev Comp Immunol. 2003;27:651-60.

41. Nakajima Y, van der Goes van Naters-Yasui A, Taylor D, Yamakawa M. Antibacterial peptide defensin is involved in midgut immunity of the soft tick, Ornithodoros moubata. Insect Mol Biol. 2002;11:611-8.

42. Oleaga A, Obolo-Mvoulouga P, Manzano-Román R, Pérez-Sánchez R. De novo assembly and analysis of midgut transcriptome of the argasid tick Ornithodoros erraticus and identification of genes differentially expressed after blood feeding. Ticks Tick Borne Dis. 2018;9:1537-54.

43. Oleaga A, Obolo-Mvoulouga P, Manzano-Román R, Pérez-Sánchez R. Functional annotation and analysis of the Ornithodoros moubata midgut genes differentially expressed after blood feeding. Ticks Tick Borne Dis. 2017;8:693-708.

44. Taylor D. Innate immunity in ticks: A review. J Acarol Soc Jpn. 2006:15:109-27.

45. Blair C, Olson K. The role of RNA interference (RNAi) in arbovirus-vector interactions. Viruses. 2015;7:820-43.

46. Forth JH, Forth LF, Lycett S, Bell-Sakyi L, Keil GM, Blome S, et al. Identification of African swine fever virus-like elements in the soft tick genome provides insights into the virus' evolution. BMC Biol. 2020;18:136.

47. Burrage TG, Lu Z, Neilan JG, Rock DL, Zsak L. African swine fever virus multigene family 360 genes affect virus replication and generalization of infection in Ornithodoros porcinus ticks. J Virol. 2004;78:2445-53.

48. Rowlands RJ, Duarte MM, Boinas F, Hutchings G, Dixon LK. The CD2v protein enhances African swine fever virus replication in the tick vector Ornithodoros erraticus. Virology. 2009;393:319-28.

49. Chastagner A, Pereira de Oliveira R, Hutet E, Le Dimna M, Paboeuf F, Lucas $P$, et al. Coding-complete genome sequence of an African wwine fever virus strain Liv13/33 isolate from experimental transmission between pigs and Ornithodoros moubata ticks. Microbiol Resour Announc 2020;9:e00185-20. https://doi.org/10.1128/MRA.00185-20.

50. Dixon LK, Chapman DAG, Netherton CL, Upton C. African swine fever virus replication and genomics. Virus Res. 2013;173:3-14.

51. Rennie L, Wilkinson PJ, Mellor PS. Transovarial transmission of African swine fever virus in the argasid tick Ornithodoros moubata. Med Vet Entomol. 2001;15:140-6.

52. Plowright $W$, Perry $C T$, Peirce MA. Transovarial infection with African swine fever virus in the argasid tick, Ornithodoros moubata porcinus Walton. Res Vet Sci. 1970;11:582-4.

53. Plowright W, Perry CT, Greig A. Sexual transmission of African swine fever virus in the tick, Ornithodoros moubata porcinus Walton. Res Vet Sci. 1974;17:106-13.

54. de la Fuente J, Antunes S, Bonnet S, Cabezas-Cruz A, Domingos AG, Estrada-Peña A, et al. Tick-pathogen interactions and vector competence: identification of molecular drivers for tick-borne diseases. Front Cell Infect Microbiol. 2017:7:114.

55. Endris RG, Hess WR. Attempted transovarial and venereal transmission of African swine fever virus by the Iberian soft tick Ornithodoros (Pavlovskyella) marocanus (Acari: Ixodoidea: Argasidae). J Med Entomol. 1994:31:373-81.

56. Haresnape JM, Wilkinson PJ. A study of African swine fever virus infected ticks (Ornithodoros moubata) collected from three villages in the ASF enzootic area of Malawi following an outbreak of the disease in domestic pigs. Epidemiol Infect. 1989;102:507-22.

57. van Gennip RGP, Drolet BS, Rozo Lopez P, Roost AJC, Boonstra J, van Rijn PA. Vector competence is strongly affected by a small deletion or point mutations in bluetongue virus. Parasites Vectors. 2019;12:470. 
58. Roundy CM, Azar SR, Rossi SL, Huang JH, Leal G, Yun R, et al. Variation in Aedes aegypti Mosquito Competence for Zika Virus Transmission. Emerg Infect Dis. 2017;23:625-32.

59. Shaw DK, Tate AT, Schneider DS, Levashina EA, Kagan JC, Pal U, et al. Vector immunity and evolutionary ecology: the harmonious dissonance. Trends Immunol. 2018;39:862-73.

60. Joy DA, Gonzalez-Ceron L, Carlton JM, Gueye A, Fay M, McCutchan TF, et al. Local adaptation and vector-mediated population structure in Plasmodium vivax malaria. Mol Biol Evol. 2008;25:1245-52.

61. Lambrechts L, Chevillon C, Albright RG, Thaisomboonsuk B, Richardson $J H$, Jarman RG, et al. Genetic specificity and potential for local adaptation between dengue viruses and mosquito vectors. BMC Evol Biol. 2009:9:160
62. Souza-Neto JA, Powell JR, Bonizzoni M. Aedes aegypti vector competence studies: A review. Infect Genet Evol. 2019;67:191-209.

63. Greay TL, Gofton AW, Paparini A, Ryan UM, Oskam CL, Irwin PJ. Recent insights into the tick microbiome gained through next-generation sequencing. Parasites Vectors. 2018;11:12

\section{Publisher's Note}

Springer Nature remains neutral with regard to jurisdictional claims in published maps and institutional affiliations.
Ready to submit your research? Choose BMC and benefit from:

- fast, convenient online submission

- thorough peer review by experienced researchers in your field

- rapid publication on acceptance

- support for research data, including large and complex data types

- gold Open Access which fosters wider collaboration and increased citations

- maximum visibility for your research: over $100 \mathrm{M}$ website views per year

At BMC, research is always in progress.

Learn more biomedcentral.com/submissions 